\title{
Activating PIK3CD Gene Mutation
}

National Cancer Institute

\section{Source}

National Cancer Institute. Activating PIK3CD Gene Mutation. NCI Thesaurus. Code C148028.

A mutation in PIK3CD, the gene encoding the p110delta catalytic subunit of PI3K, that results in induction of PI3K signaling. Mutations of this type are associated with immunodeficiency 14 (PASLI: activated PI3k-delta syndrome, p110-delta-activating mutation causing senescent T-cells, lymphadenopathy, and immunodeficiency). 